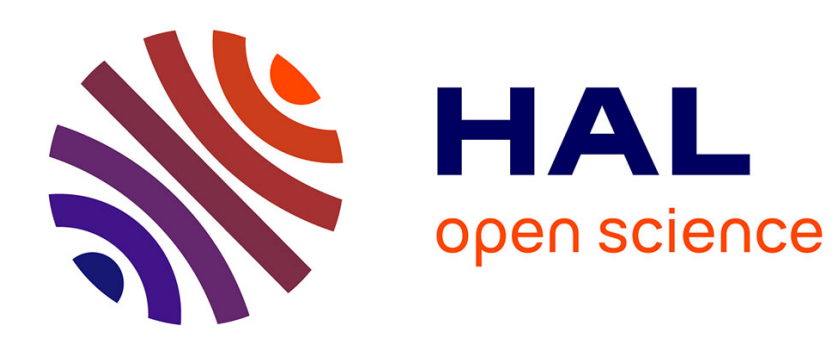

\title{
Gender Differences in the Relational and Collective Bases for Trust
}

\author{
William W. Maddux, Marilynn B. Brewer
}

\section{To cite this version:}

William W. Maddux, Marilynn B. Brewer. Gender Differences in the Relational and Collective Bases for Trust. Group Processes and Intergroup Relations, 2005, 8 (2), pp.159-171. 10.1177/1368430205051065 . hal-00571600

\section{HAL Id: hal-00571600 \\ https://hal.science/hal-00571600}

Submitted on 1 Mar 2011

HAL is a multi-disciplinary open access archive for the deposit and dissemination of scientific research documents, whether they are published or not. The documents may come from teaching and research institutions in France or abroad, or from public or private research centers.
L'archive ouverte pluridisciplinaire HAL, est destinée au dépôt et à la diffusion de documents scientifiques de niveau recherche, publiés ou non, émanant des établissements d'enseignement et de recherche français ou étrangers, des laboratoires publics ou privés. 


\title{
Gender Differences in the Relational and Collective Bases for Trust
}

\author{
William W. Maddux \\ Northwestern University \\ Marilynn B. Brewer \\ The Ohio State University
}

\begin{abstract}
A variety of research suggests that men and women differ in their interdependent orientation: whereas women tend to be more relationally interdependent, men tend to be more collectively interdependent (e.g. Gabriel \& Gardner, 1999). The current study sought to investigate differences in interdependence within the domain of trust. In particular, the authors predicted that men would tend to trust individuals based on whether or not they shared group memberships. On the other hand, women were predicted to trust those who shared direct or indirect relationship connections. Results from an online trust-dilemma game supported these predictions. Implications for our understanding of the impact of gender on social identity and self-representation are discussed.
\end{abstract}

KEYWORDS gender, self-construal, social identity, trust

MEN and women differ. Such a statement would strike most people as intuitively obvious. That gender differences are believed to be relatively ubiquitous is underscored by the fact that the topic is well-picked fodder for popular culture and the entertainment media; gender conflicts are often explored in sitcoms, reality TV shows, comedic and dramatic movies, and stand-up comedy. However, results from psychological research indicate that there may be some basis for the widespread belief that men and women differ. Empirical evidence suggests that gender differences exist across a variety of psychological domains, including emotion, cognition, behavior, and language use (for a review, see Deaux, 1998).
Although it is clear that gender impacts a variety of domains, recent research is beginning to uncover some of the specific psychological foundations of how men and women differ. One primary area of interest is self-representation. For example, in a review of the literature Cross and Madson (1997) proposed that a number of

\section{Author's note}

Address correspondence to William W.

Maddux, Dispute Resolution Research Center, Kellogg School of Management, Northwestern University, 2001 Sheridan Rd., Evanston, IL 60208, USA

[email: w-maddux@kellogg.northwestern.edu] 
previously documented gender differences could be explained in terms of differences in self-construals: while men may have more of an independent construal of self, women may have more of an interdependent construal of self. Within this framework, an independent self-construal accentuates self-related features and excludes the influence of others in the self-schema, whereas an interdependent selfconstrual represents inclusion of others in the self (Aron, Aron, \& Smollan, 1992; Brewer \& Gardner, 1996; Markus \& Kitayama, 1991).

Cross and Madson (1997) reviewed a variety of evidence in support of this hypothesis. For example, they noted that while women often describe themselves more in terms of relationships with others, men have a stronger tendency to describe themselves in terms of separateness from others (e.g. Pratt, Prancer, Hunsberger, \& Manchester, 1990). In addition, women tend to rate themselves more highly on self-related dimensions concerning interdependence, while men rate themselves higher on independent dimensions (e.g. Zuckerman, 1985). Finally, Cross and Madson cited evidence that in terms of conversational norms, women prefer talking about relationships, while men prefer discussing less personal topics such as sports and politics (e.g. Aries \& Johnson, 1983).

Additional research has extended the idea of gender differences in self-construals from the individual level to the collective level. For example, Baumeister and Sommer (1997) proposed that a sense of belongingness is a fundamental human need (cf. Baumeister \& Leary, 1995), and thus having a sense of connectedness to others is essential psychologically for both men and women. However, Baumeister and Sommer proposed that men and women differ in the way in which they satisfy this need. While men may indeed be more independent than women, at the interdependent level men may also place a greater importance on group memberships and large collectives. In other words, in terms of the way in which people feel a sense of interdependence with others, women may be more relationally oriented, men may be more collectively oriented (Baumeister \& Sommer, 1997).
Gabriel and Gardner (1999) obtained a variety of support for the existence of gender differences in relational versus collective interdependence. In one study involving a diaryreading paradigm, women showed better selective memory for relational items in the diary, while men showed better memory for collective items. In another study, women were found to be more likely to put their own personal desires aside for a friend, while men were more likely to sacrifice for a group (Gabriel \& Gardner, 1999). In addition, similar gender differences have been found to impact the subjective importance of different types of groups (Seeley, Gardner, Pennington, \& Gabriel, 2003). For women, group importance is mainly determined by the degree to which the group fulfills relational needs, while men place a greater importance on the collective identity that groups offer. ${ }^{1}$

The existence of gender differences in interdependence is consistent with the idea that there are two distinct types of selfrepresentations that operate in social contexts. More specifically, Brewer and Gardner (1996) postulated that there are two different levels of 'social selves': a relational self and a collective self. In this framework the relational self concerns self-representations derived from one's relationships with specific other people ('I am a father'). In terms of gender research, the idea of the relational self corresponds closely to Cross and Madson's conception of the interdependent self-construal that predominates for women. On the other hand, the collective self is constructed from one's identity as derived from memberships in groups ('I am a Cubs fan'). This idea of the collective self seems to correspond more closely to the collective-interdependent nature of men. Thus, in terms of Brewer and Gardner's (1996) conception of social selves, the relational self seems the more salient type of social self for women, while the collective self is the more salient type of social self for men.

It is important to point out that the above research has focused primarily on the selfconstrual of women in Western cultures (i.e. the United States, Canada, Australia, Western Europe). Although interdependent self-construals 
have also been shown to be particularly salient for individuals from East Asian cultures (Markus $\&$ Kitayama, 1991), to date there is little research on the similarities of the interdependent selfconstruals of Western and East Asian women; however, there is evidence for some overlap, specifically concerning emotional relatedness with significant others (Kashima et al., 1995). However, other areas of potential overlap remain unexplored, and it remains to be seen whether universal gender differences exist in other psychological domains relevant to self-construals.

The way in which one construes the self is of importance primarily because of the tremendous influence that the self has on a host of psychological processes. The self has been shown to be a major organizing factor for the type of thoughts we have about ourselves and others, what information we pay attention to and what information we ignore, how we feel about and evaluate ourselves, with whom we choose to compare ourselves, and how we choose to present ourselves to others (for a review, see Baumeister, 1998). Thus, whether one's dominant self-construal is independent or interdependent, or whether one's salient social self tends to involve relationships or collective memberships, these construals of self have the potential to have a great impact on how we deal with almost any type of situation in daily life.

Overall, then, there is growing evidence that gender differences impact the way in which people feel a sense of interdependence with others and define their ingroups. Compared to men, women place more emphasis on relationships and interpersonal connections, while men are more likely to emphasize more depersonalized group memberships and the importance of group identity. Importantly, these gender differences in interdependence have been shown to impact a variety of psychological variables, including self-representation, selective memory, and group attachment.

\section{Consequences for depersonalized trust}

If there are indeed reliable gender differences in relational and collective interdependence, these differences should also manifest themselves in situations where men and women must decide whether or not to trust other people, particularly strangers. Although the concept of trust has always been an integral aspect of research in social psychology, there has been a recent resurgence of interest in trust as a central psychological construct. Of particular interest to social psychologists is the role of trust in contexts and institutions where participants must decide whether or not to cooperate with others with whom they have no personal knowledge or history of interpersonal relationship. Such 'depersonalized trust' is essential for the creation and maintenance of many forms of economic exchange, organizations, and social and political institutions.

There are at least two possible bases for trusting strangers. First of all, shared category membership may become a basis for depersonalized trust (Brewer, 1981; Buchan, Croson, \& Dawes, 2002; Macy \& Skvoretz, 1998; Yamagishi \& Kiyonari, 2000). As a consequence of shifting psychologically from the personal to the collective level of identity, one may be less likely to distinguish the interests of other ingroup members from those of oneself, leading to increasing trust toward fellow ingroup members. Hence, simply knowing that an otherwise unknown person is a member of a salient ingroup may be sufficient to engender trust as a default assumption. A second route involves sharing a network of interpersonal relations with others. Individuals may trust others if they know (or believe) that they are directly or indirectly connected to each other through mutual friendships or acquaintances (Coleman, 1990). In other words, we may trust a stranger if we believe that the person is potentially a member of a generalized exchange network of relationships (Yamagishi \& Yamagishi, 1994).

Given these distinctions in the basis for depersonalized trust, if men are more collectively interdependent than women, men should be more likely to trust individuals who share ingroup membership, regardless of personal relationship connections. By contrast, if women are more relationally interdependent, then women should tend to trust individuals who are 
likely to share a direct or indirect network of relationships, and ingroup/outgroup distinctions should be less important. We tested these predictions in an online, real-time money allocation game that provided an engrossing, meaningful trust-related situation.

\section{The present research}

The current study employed a modified form of the allocator/dictator game devised by Kiyonari and Yamagishi (1999). In this game, participants make online decisions about whether to accept a 'sure-thing' payment of US\$3 from the experimenter, or an unknown allocation from a stranger (who had ostensibly been given US $\$ 11$ to distribute as he or she wished). Since actual monetary payments were involved and participants were told that their payment at the end of the experiment depended on the outcomes of their decisions, this paradigm provides a compelling test of participants' willingness to place faith in a stranger based on minimal information about another person's social group memberships or potential relationship connections.

This paradigm has previously proven to be a reliable method for investigating cross-cultural differences in depersonalized trust (Yuki, Maddux, Brewer, \& Takemura, 2005). For example, Yuki and colleagues (2005) showed that although American participants trusted ingroup members more than outgroup members (regardless of the presence of crossgroup relationship connections), Japanese participants showed no differences in trust toward ingroup members and outgroup members who had a potential cross-group relationship connection. Since a variety of research has demonstrated that Japanese are more relationally oriented than Americans (e.g. Markus \& Kitayama, 1991; Yuki, 2003), the current study sought to explore similar gender effects in this paradigm. Although no gender differences emerged in Yuki et al.'s (2005) American sample, we hypothesized that priming both men and women with interdependence could potentially activate pre-existing gender differences in an American sample. Previous research has sometimes found it necessary to prime the interdependent self in order to induce interdependent cognition for American participants (Brewer \& Gardner, 1996).

Thus, in the current research we first presented American men and women with one of two types of primes designed to activate interdependence with others; in other words, the primes served to activate the 'social self'. ${ }^{2}$ Subsequently men and women then participated in an online, allocator/dictator game that measured how much they trusted a variety of targets whose personal identity was unknown. One target was an ingroup target, identified as a student at the same university as the participant. A second target, representing a crossgroup relationship target, was identified as a student at an outgroup university where the participant had earlier indicated that he or she had an acquaintance. A third target, representing a basic outgroup target, was identified as a student at a university where the participant did not indicate knowing anyone. In reality, all targets were fictitious and were used as part of the cover story to create a realistic setting for trust decisions. Participants performed one trial with each target, the order of which was randomly determined.

Based on the collective-interdependent nature of men, we predicted that men would show the highest level of trust for the ingroup target, and significantly less trust for both outgroup targets. Based on the relationshipinterdependent nature of women, we predicted that the presence of a cross-group relationship would increase trust for an outgroup member more for women than for men. We expected women to show high levels of trust for both the ingroup and cross-group relationship target, and significantly less trust for the outgroup target. Thus, our paradigm had a 2 (version of interdependent prime) $\times 2$ (participant gender) $\times 3$ (type of target) mixed factorial design, with prime and gender as betweensubjects variables, and type of target as a withinsubjects variable. 


\section{Method}

\section{Participants}

Participants were 147 students (79 males, 68 females) at Ohio State University. All participants were citizens of the United States. Participants were recruited from the introductory psychology subject pool, and voluntarily signed up in return for partial course credit and a monetary payment. The experimental requirements indicated that in order to be eligible for the experiment, participants had to have acquaintances at other Big Ten universities (the conference of universities that includes Ohio State University). Four participants (two males and two females) gave at least one incorrect response on one of the manipulation checks, and their data were excluded from further analysis. This left the data from 143 participants (77 males, 66 females) for formal analysis.

\section{Priming materials}

Participants were randomly assigned to read one of two scenarios designed to prime interdependence. One scenario involved a story about playing a round of miniature golf with a group of friends, and a second scenario involved a story about attending a baseball game. For both scenarios, participants were instructed to circle all the pronouns in the story, and then were asked several questions about the content of the story (e.g. what was the story about?, what type of words did you circle?) as part of the cover story that the experiment concerned 'verbal ability and memory'. Both versions contained 21 plural pronouns (us, we, our). These scenarios were designed to prime an interdependent frame of mind (Brewer \& Gardner, 1996).

\section{Procedure}

Participants were shown into the lab and seated at a computer terminal. A female experimenter explained that they would be participating in two separate experiments. Participants were instructed to complete the first paper-andpencil experimental packet, after which they were to turn on their computers to complete the second experiment on judgment and decision-making. The experimenter then told participants they were free to begin, at which time she left the room.

Participants then completed one of the two independent priming packets as part of an experiment on 'verbal ability and memory', which was in actuality the priming phase of the experiment. Following this priming phase, participants turned on their computers and began the main trust experiment. The instructions on the screen explained that the second study was an online decision-making game in which they would be interacting with other participants in a real-time, money allocation situation. It was explained that participants would be paid for their participation in the experiment, as well as receiving partial course credit. Participants were initially asked to enter the names and universities of all acquaintances they had at Big Ten universities. The computer then paused for a few seconds, and as part of the cover story, the instructions indicated that the computer was connecting to an online network. In reality, the computer did not connect to any network, and was simply programmed to wait 20 seconds before continuing.

The main instructions then began by reiterating that the experiment was concerned with judgment and decision-making, and that it was being conducted in conjunction with students from other universities. Participants were told that some conditions involved making decisions with other Ohio State students, while other conditions involved decisions with students from other universities, who were performing the experiment at the same time, but in a different location. The decision task involved dividing a fixed sum of money (US\$11) between two paired individuals. Participants were told that one person would be randomly assigned the role of the allocator (the person who decides how much to give to each person), and the other would be assigned the role of the recipient. The allocator's task was ostensibly to decide how much of the money he/she would keep for himself/herself, and how much to give to the other participant. There were no rules for the monetary division, and the allocator was supposedly allowed to divide up the money however he/she chose. Participants were told 
that their final payment was to be based on the amount of money they received (or kept) during this decision-making task. The instructions indicated that each participant would only know one thing about the other person-the university he or she was attending.

The computer then paused and the instructions indicated that it was randomly being determined which person would play the role of allocator, and which person would be the recipient. However, the computer always assigned participants to the role of the recipient, while the ficticious partner was always assigned the role of allocator. The instructions explained that the recipient had to choose between two options in the game: the exit option of a 'sure thing' (receiving US\$3 from the experimenter), or the option of taking whatever money the allocator decided to give him/her. The instructions also indicated that this choice had to be made before the other partner's choice was disclosed. In reality, of course, the allocator never made a decision. We were simply interested in whether participants chose to take this unknown allocation of money, or whether they took the sure thing. Thus, the decision to accept the allocator's allotment involved giving up $\$ 3$, with the risk of winding up with less (or no) money allocation. ${ }^{3}$

The computer paused while the instructions indicated that the participant's partner was now being chosen, then randomly selected either an ingroup target, a potential relationship target, or an outgroup target. If the computer selected the ingroup target, the instructions indicated that the allocator was a student from Ohio State. If the potential relationship target was chosen, the computer indicated that the allocator was from another university, but that our records indicated that this was a university at which the participant had an acquaintance. If the outgroup target was chosen, the computer said that the allocator was from another university, and that our records indicated that the participant did not know anyone at that university. ${ }^{4}$

Once the allocator target was chosen and identified, participants were instructed to make their choice, either the sure-thing payment of
$\$ 3$, or taking whatever amount the allocator had given them. This yes/no decision was our behavioral measure of whether participants trusted the target individuals or not. Following the decision trial, participants were asked three follow-up questions. First, participants were asked, 'To what extent did you trust the allocator to make a decision that was favorable to you?' (1 did not trust at all, 5 completely trusted). Next participants were asked, 'How much money do you think the allocator gave you?' Participants were asked to enter an amount in US dollars, from $\$ 0$ to $\$ 11$. Finally, as a manipulation check to insure participants were paying attention to the instructions, participants were asked to 'please indicate what university the previous allocator was from'. The computer then provided two options: Ohio State or another university. Participants were asked to mark the correct response.

Following completion of the first trial, the instructions stated that there were to be two more decision trials. Participants were told that their final payment would be based on the decision made on one trial, and that this critical trial would be randomly selected at the end of the experimental session. This was done in order to motivate participants to maximize their possible reward for each trial independently of the others. The choice paradigm was then repeated over two more trials. All participants performed one trial for each target type, i.e. once with an ingroup target, once with a potential relationship target, and once with an outgroup target, with the order varied randomly across participants. Following each decision trial, participants were again asked to indicate their trust for the target, the amount of money allocated by the target, and the allocator's university.

After these questions, the computer indicated that one trial would be randomly selected and that participants would receive payment based on the outcome of that trial. However, the computer always chose the second decision trial as a basis for payment. Participants were paid the $\$ 3$ if they had chosen the sure-thing option on trial 2 , and they were paid $\$ 4$ if they had chosen to forgo the sure thing and take the 
partner's allocation. Following payment, participants were probed for suspicions about the cover story, debriefed about the true nature of the experiment, and thanked for taking part.

\section{Results and discussion}

\section{Online decisions}

Initial analyses were carried out on participants' decisions for each of the three different target allocators. These decisions were subjected to several successive nonparametric analyses to compare targets. ${ }^{5}$ An initial Cochran's $Q$ test indicated that participants' decisions whether to accept the allocator's division of the money differed significantly across the three target conditions, with participants taking the ingroup target's allocation $89 \%$ of the time, the relationship target's allocation $83 \%$ of the time, and outgroup target's allocation $75 \%$ of the time $(Q(2)=$ $20.15, p<.001)$.

Individual McNemar tests on the comparisons for specific targets showed that compared to the ingroup target, participants were less likely to accept the allocator's decision and more likely to take the sure thing for the relationship target $\left(\chi^{2}(143)=4.53, p=.035\right)$, and for the outgroup target $\left(\chi^{2}(143)=16.69\right.$, $p<.001)$. In addition, participants were also significantly less likely to accept the allocator's decision and more likely to take the sure thing for the outgroup target compared to the relationship target $\left(\chi^{2}(143)=4.70, p=.030\right)$.

These results indicate that participants were most willing to give up the sure-thing option when the target was an ingroup member, less likely with the potential relationship target, and least likely when the target was an outgroup member. Thus, willingness to forgo a sure thing in favor of an allocation from an unknown person was clearly sensitive to both types of information; that is, whether the target was an ingroup or outgroup member and whether the target was potentially part of an indirect relationship network. However, contrary to expectations, this decision was not affected by gender; men and women did not differ in their likelihood of forgoing the sure thing option in favor of the allocator's decision for any of the three targets (ingroup target: men $=88 \%$, women $=90 \%$; relationship target: men $=84 \%$, women $=82 \%$; outgroup target: men $=73 \%$, women $=77 \%$, all $p \mathrm{~s}>.22$ ).

However, results on the trust ratings and expectations of monetary awards did support predictions. A 2 (version of interdependent prime) $\times 2$ (gender) $\times 3$ (target type), mixedfactorial analysis of variance (ANOVA) was conducted on trust ratings, with target as a within-subjects variable, and participant gender and type of prime as between-subjects variables. The results indicated a main effect for target type $\left(F(2,496)=33.70, p<.001, \eta^{2}=.328\right)$, such that most trust was shown toward the ingroup member, and least trust toward the outgroup member, consistent with the pattern of choice decisions. However, this main effect was qualified by a significant two-way interaction between target type and gender $(F(2,278)=4.80, p=$ $\left..009, \eta^{2}=.033\right){ }^{6}$

A series of pair-wise comparisons was carried out to decompose the differences in trust toward specific targets. Within-gender comparisons indicated that men trusted the ingroup target significantly more than both the potential relationship target $(F(1,76)=33.61$, $\left.p<.001, \eta^{2}=.301\right)$, and the outgroup target $\left(F(1,76)=44.45, p<.001, \eta^{2}=.353\right)$, while there was no significant difference in trust for the potential relationship target compared to the outgroup target $(p>.46)$. Thus, men trusted ingroup members more than outgroup members.

However, for women, trust was greater toward the ingroup target than the outgroup target $\left(F(1,65)=24.91, p<.001, \eta^{2}=.277\right)$, but women also trusted the potential relationship target more than the outgroup target $(F(1,65)$ $\left.=8.97, p=.004, \eta^{2}=.121\right)$. There was no difference in trust for the ingroup target and potential relationship target for women $(p>.25)$. Thus, women trusted the ingroup target and the potential relationship target more than the outgroup target.

Cross-gender comparisons indicated no differences in trust toward the ingroup and outgroup targets $(p s>21)$. However, women 
were significantly more trusting of the potential relationship target than were men $(F(1,141)=$ $\left.11.16, p=.001, \eta^{2}=.073\right)$. Thus, these results support our predictions that the presence of a cross-group relationship would increase trust more for men than for women. While there were no gender differences in trust toward ingroup and outgroup members, women trusted the potential relationship target significantly more than did men. In fact, for women, there was no significant difference in trust toward the ingroup member and the potential relationship target (see Figure 1).

We also analyzed the amount of money participants expected to receive from each of the three allocators. Results of these analyses mirrored those of allocator trust (see Figure 2). A 2 (version of interdependent prime) $\times 2$ (gender) $\times 3$ (target type), mixed-factorial
ANOVA was conducted on these data, with target as a within-subjects variable, and participant gender and type of prime as betweensubjects factors. A significant main effect emerged for target $(F(2,278)=11.94, p<.001$, $\left.\eta^{2}=.079\right)$, such that the most money was expected from the ingroup member, and the least from the outgroup member. This main effect was qualified by a significant two-way interaction between target type and gender $\left(F(2,278)=3.37, p=.036, \eta^{2}=.024\right)$, indicating that the expected money differed depending on participants' gender. No differences emerged based on type of prime, so further analyses were again collapsed across primes.

As with the trust analyses, pair-wise comparisons indicated that men expected more money from the ingroup target than the potential relationship target $(F(1,75)=11.45, p=.001$,

\section{Amount of Trust}

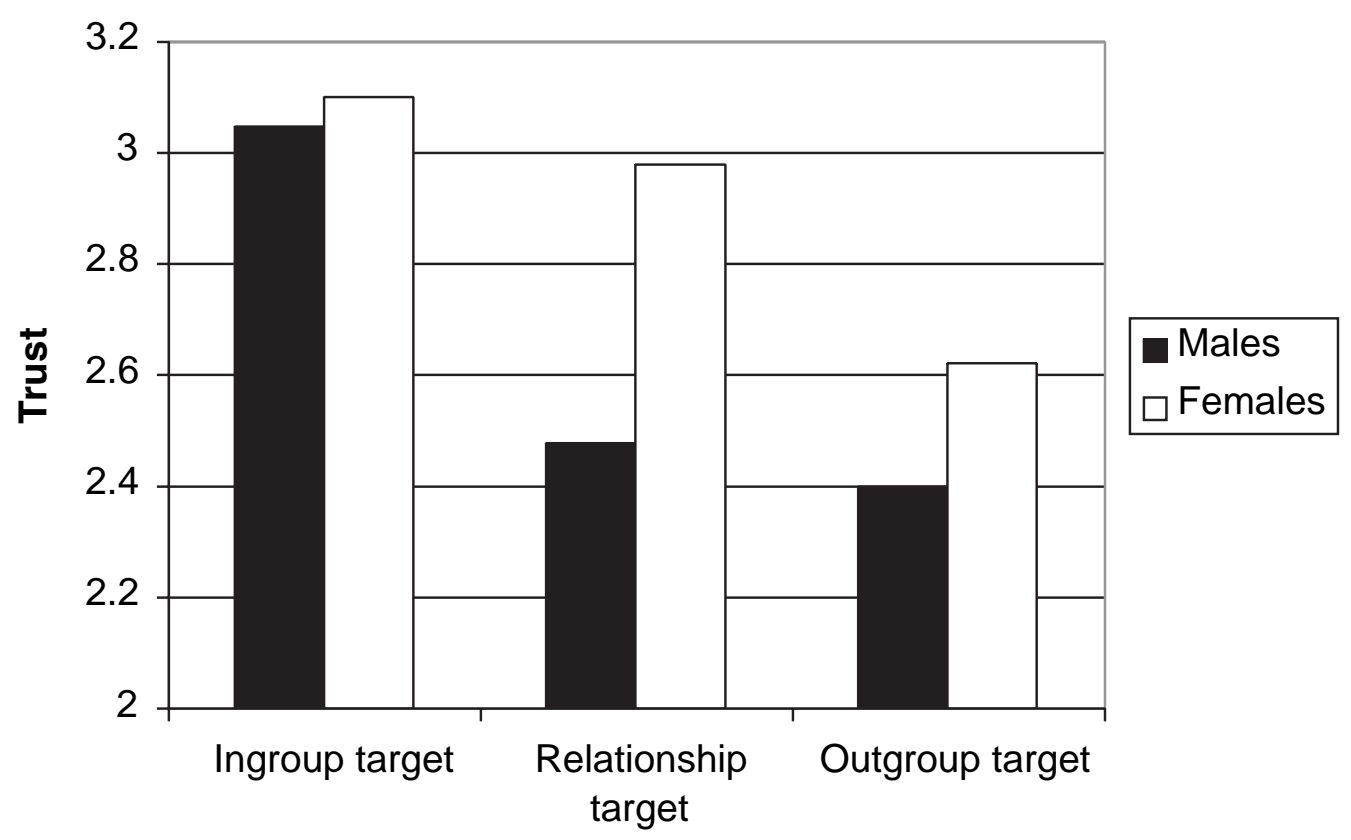

\section{Allocator}

Figure 1. Amount of trust per target as a function of relationship type and participant gender (1 don't trust at all, 5 trust completely). 
$\left.\eta^{2}=.132\right)$, and more money from the ingroup target than the outgroup target $(F(1,75)=$ $\left.23.50, p<.001, \eta^{2}=.239\right)$. There was no difference between the potential relationship target and the outgroup target $(p>.26)$. For women, expected money did not differ between the ingroup target and the potential relationship target $(p>.84)$. However, a difference in expected money did emerge between the ingroup target and the outgroup target $(F(1$, $\left.64)=10.62, p=.002, \eta^{2}=.142\right)$, and there was a marginal difference between the potential relationship target and the outgroup target $\left(F(1,64)=3.96, p=.051, \eta^{2}=.058\right)$.

Finally, cross-gender comparisons of the expectation ratings indicated no differences between men and women in trust toward the ingroup targets $(p>.98)$, although women expected marginally more money from the outgroup target than men $\operatorname{did}(F(1,141)=3.15$, $\left.p=.078, \eta^{2}=.022\right)$. However, as predicted, women also expected significantly more money from the potential relationship target than men $\left(F(1,141)=6.72, p=.011, \eta^{2}=.046\right)$. These results were essentially identical to those on the trust rating measure.

To summarize, although the predicted differences did not emerge on our behavioral measure, self-report measures did reveal the expected pattern of trust. Compared to men, women were significantly more likely to trust an outgroup member who shared a potential crossgroup relationship connection. In addition, for women the level of trust toward an ingroup member and toward an outgroup member with a cross-group relationship connection did not differ; however, men trusted ingroup members significantly more than outgroup members regardless of cross-group relationship connections. This same pattern also appeared on the

\section{Expected Money}

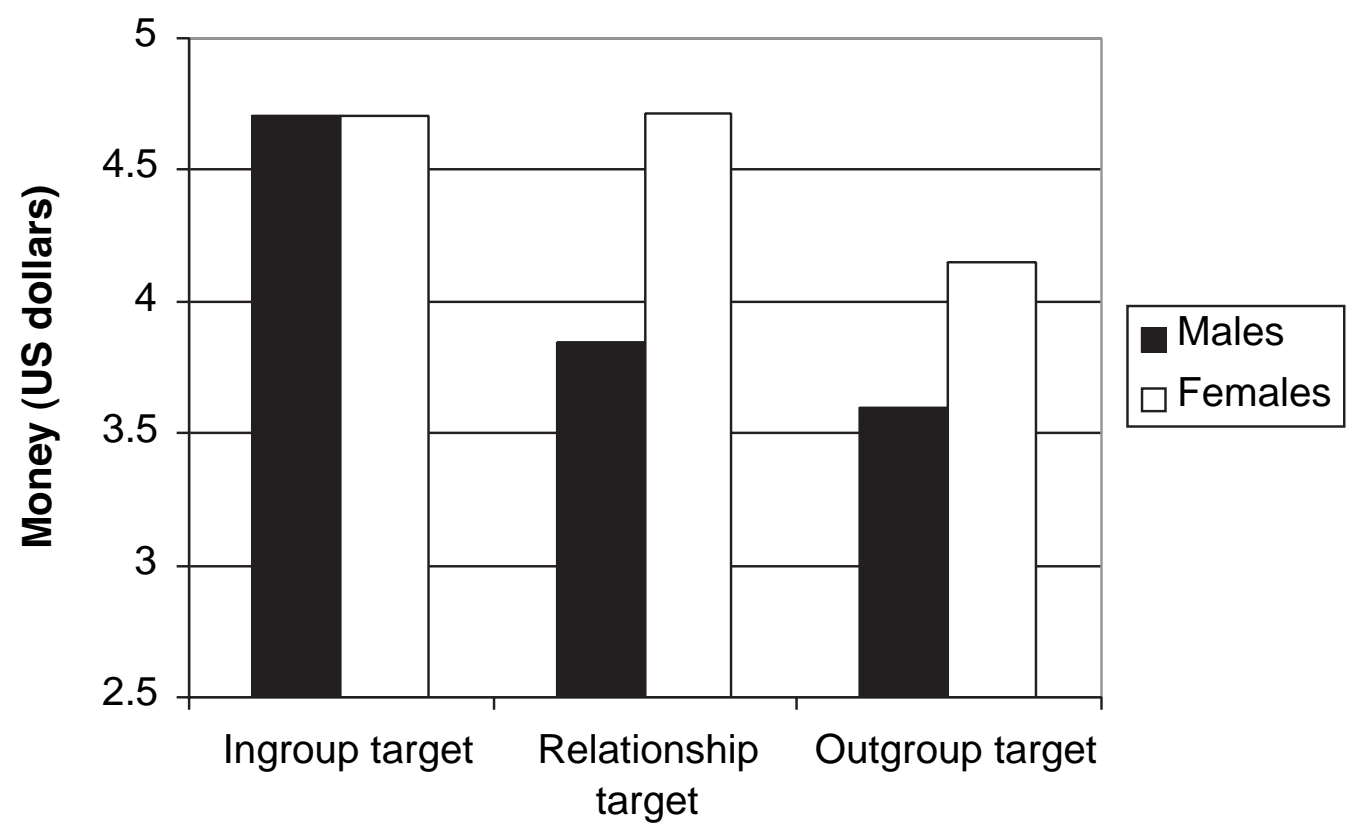

Allocator

Figure 2. Expected money per target as a function of relationship type and participant gender (US\$0 to US\$11). 
measure of the amount of money participants expected from each target. Men expected significantly more money from ingroup members than from outgroup members. Women expected no differences in money from an ingroup member and an outgroup member with a potential cross-group relationship, but significantly less money was expected when the outgroup member had no relationship connections. These results indicate that trust for men was largely dependent on sharing group memberships, while trust for women depended more on sharing relationship links.

\section{General discussion}

The main goal of the present research was to investigate the impact of gender differences in interdependence within the domain of trust. The results from an online, allocator/dictator trust game largely supported our predictions based on the idea that women are more relationally interdependent and men are more collectively interdependent. For men, trust (and expected money) was highest for ingroup members compared to outgroup members. However, the presence of a potential crossgroup relationship increased trust (and expected money) more for women than for men. This potential cross-group relationship connection was sufficiently meaningful for women that no differences in trust emerged toward the ingroup target and the potential relationship target. The fact that even a potential relationship connection with an outgroup member increased trust to the level of an ingroup member is striking evidence that relational interdependence is particularly salient for women. Thus, cross-group relationships had a greater impact on trust for women, while the categorical distinction between ingroup and outgroup was more important for men.

Although the predicted pattern of trust did not emerge on our behavioral measure-the decision to take or forgo a sure-thing option of $\$ 3$-we believe this is not overly problematic, primarily because such a decision is a relatively indirect measure of trust. In the current paradigm participants were instructed and encouraged to maximize their monetary reward for the online decisions. Thus, not only was trust not made particularly salient for the decision trials, but any gender differences in trust may have been masked by other influences, particularly differences in preferences for risky decision-making.

For example, a variety of research suggests that men are more likely, across a variety of domains, to make more risky decisions than women (e.g. Sorentino, Hewitt, \& Raso-Knott, 1992; Weber, Blais, \& Betz, 2003; for a review, see Byrnes, Miller, \& Schafer, 1999). Thus, although women may have actually trusted the relationship target more than men (as evidenced on the self-report measures), women's decreased tolerance for risky decisions may have inflated their preference for choosing the safer, sure thing option, eliminating any gender differences that may have existed. This explanation is supported by the fact that compared to men, women actually expected to receive significantly more money from the potential relationship allocator, but nevertheless did not decide to give up the sure thing more often than men. Thus, it is quite likely that women simply had a higher threshold for deciding to give up the safe option.

In addition, we would argue that the selfreport measures of trust and expected money are more reliable than the behavioral measure because they directly and explicitly assess participants' level of trust toward targets, as well as their expectations for others' behaviors. The fact that these self-report measures replicated earlier research using the same paradigm (Yuki et al., 2005) is additional evidence for their reliability and validity. Finally, it is important to note that the allocator/dictator game was an effective way to create an engaging and meaningful situation in which to assess trust and expected monetary allocations toward a variety of targets-a paradigm likely to be more diagnostic of trust compared to paradigms where participants are merely asked to imagine how they would respond.

Overall, the current results offer support for different types of interdependence for men and women. The research also extends previous 
findings into a new domain, that of trust. Decisions concerning whether or not to trust strangers are important in a variety of areas of everyday life, including the creation and maintenance of many forms of economic exchange, social relationships, and political institutions. In social exchange situations, it is important to know what types of information or minimal social cues will lead one person to expect to be able to trust another. To the extent that there are cultural and gender differences in the bases for depersonalized trust, coordination of trust may require an understanding of those differences.

Our results also offer additional evidence for the existence of two distinct types of social selves, a relational self and a collective self (Brewer \& Gardner, 1996). While the relational self refers to the inclusion of relationships within one's self-representation, the collective self concerns the inclusion of group identity and group memberships as part of the self. We assume that both men and women need and value both relational connections with others and group memberships, but that they differ in which level of social self is most salient or accessible. That trust for women seems to depend largely on direct and indirect relationships is consistent with the idea that the relational self is the predominant type of social self for women. The finding that trust for men was mostly dependent on the categorical, ingroup/ outgroup distinction suggests that the collective self may be the more salient type of social self for men.

It is likely that gender differences in the social self are influenced by socialization norms and culturally specific role expectations as individuals grow up (cf. Eagly, 1987). For example, there is evidence that among children and adolescents, female play-groups tend to emphasize interpersonal interactions, while male playgroups emphasize teams and large groups (e.g. Benenson, Apostoleris, \& Parnass, 1997). Although most research has focused on socialization and self-construal of women in the West, differential socialization of males and females may be very similar across cultures. In a large study involving respondents from five different countries, Kashima et al. (1995) found a pancultural gender difference in relational interdependence. Across all cultures, women scored higher than men on a scale assessing emotional relatedness of the self with significant others, providing support for the idea that gender differences in interdependence may be relatively ubiquitous, and not specific to Western cultures.

It is also noteworthy that the current results show an interesting parallel with results from our previous research concerning cross-cultural differences in depersonalized trust (Yuki et al., 2005). As was true for women in the current study, in our earlier experiments the presence of a potential cross-group relationship increased trust more for Japanese than for Americans within the same allocator/dictator paradigm used in the present study. This earlier study showed no gender differences within the Japanese sample, although there was a nonsignificant trend $(p=.18)$, with Japanese women showing more trust toward the relational target than Japanese men.

However, despite the parallel between cultural differences and gender differences in self-construal, it is not necessarily the case that effects of culture and gender on trust stem from the same underlying processes. After all, the meaning of interpersonal relationships is culturally embedded, and it is possible that Asian and Western women may differ qualitatively in the way in which they are interdependent. Although emotional interdependence with significant others has been shown to be similar across cultures (Kashima et al., 1995), it remains to be seen how much overlap there is in interdependent self-construal in other relevant domains, such as cognition, motivation, and behavior.

Thus, although East Asians (both men and women) and American women have been shown to be sensitive to cross-group relationships as a basis for trusting a stranger, this trust may reflect very different expectations or assumptions in the two cases. Future research should continue to explore the nature of culture and gender differences in relational and collective interdependence, and the way in 
which the foundations of interdependent orientation may be similar or different depending on gender and culture.

\section{Notes}

1. It should be noted that some research has demonstrated main effects for the primary importance of relational interdependence over collective interdependence across genders (Gabriel \& Gardner, 1999). In addition, other results have shown that men are concerned with both relational and collective interdependence (e.g. Seeley et al., 2003). However, we have maintained the current terminology emphasizing relative differences between genders since findings consistently show an interaction between gender and type of interdependence, with women being more relationally and less collectively oriented than men.

2. Two types of primes were used in order to be able to generalize our findings across primes regardless of idiosyncratic content of each particular prime. Participants received only one of the two primes.

3. It is important to note that the allocator supposedly only knew the university identity of the recipient; he/she was not supposed to be aware of the sure-thing option (Kiyonari \& Yamagishi, 1999).

4. For both outgroup targets, the specific university membership of the allocator was not mentioned in order to avoid the effects of specific stereotypes unique to each university.

5. Results of the online decisions were the same regardless of the type of prime participants were given, so reported analyses are collapsed across both types of primes.

6. There was also a marginally significant three-way interaction involving target, gender, and version of the prime. The gender $\times$ target effect was somewhat stronger for participants who had seen the golfing prime story than the baseball game story. Nonetheless we have collapsed across the versions of the prime because the overall interaction pattern was the same for both.

\section{Acknowledgment}

This research was supported by a grant from the Russell Sage Foundation.

\section{References}

Aries, E. J., \& Johnson, F. L. (1983). Close friendships in adulthood: Conversational content between same-sex friends. Sex Roles, 9, 1183-1196.

Aron, A., Aron, E. N., \& Smollan, D. (1992). Inclusion of other in the self scale and the structure of interpersonal closeness. Journal of Personality and Social Psychology, 63, 596-612.

Baumeister, R. F. (1998). The self. In D. Gilbert, S. Fiske, \& G. Lindzey (Eds.), The handbook of social psychology (pp. 680-740). New York: Oxford University Press.

Baumeister, R. F., \& Leary, M. R. (1995). The need to belong: Desire for interpersonal attachments as a fundamental human motivation. Psychological Bulletin, 117, 497-529.

Baumeister, R. F., \& Sommer, K. L. (1997). What do men want? Gender differences and two spheres of belongingness: Comment on Cross and Madson. Psychological Bulletin, 122, 38-44.

Benenson, J. F., Apostoleris, N. H., \& Parnass, J. (1997). Age and sex differences in dyadic and group interaction. Developmental Psychology, 33, 538-543.

Brewer, M. B. (1981). Ethnocentrism and its role in interpersonal trust. In M. Brewer \& B. Collins (Eds.), Scientific inquiry and the social sciences (pp. 345-360). San Francisco: Jossey-Bass.

Brewer, M. B., \& Gardner, W. (1996). Who is this 'we'? Levels of collective identity and self-representations. Journal of Personality and Social Psychology, 71, 83-93.

Buchan, N. R., Croson, R. T. A., \& Dawes, R. M. (2002). Swift neighbors and persistent strangers: A cross-cultural investigation of trust and reciprocity in social exchange. American Journal of Sociology, 108, 168-206.

Byrnes, J. P., Miller, D. C., \& Schafer, W. D. (1999). Gender differences in risk taking: A meta-analysis. Psychological Bulletin, 125, 367-383.

Coleman, J. S. (1990). The foundation of social theory. Cambridge, MA: Harvard University Press.

Cross, S. E., \& Madson, L. (1997). Models of the self: Self-construals and gender. Psychological Bulletin, 122, 5-37.

Deaux, K. (1998). Gender. In D. Gilbert, S. Fiske, \& G. Lindsay (Eds.) The handbook of social psychology (Vol. 1, 4th ed.), pp. 788-828. New York: McGraw-Hill.

Eagly, A. H. (1987). Sex differences in social behavior: A social-role interpretation. Hillsdale, NJ: Erlbaum. 
Gabriel, S., \& Gardner, W. L. (1999). Are there 'his' and 'hers' types of interdependence? The implications of gender differences in collective versus relational interdependence for affect, behavior, and cognition. Journal of Personality and Social Psychology, 77, 642-655.

Kashima, Y., Yamaguchi, S., Kim, U., Choi, S., Gelfand, M.J., \& Yuki, M. (1995). Culture, gender, and self: A perspective from individualism-collectivism research. Journal of Personality and Social Psychology, 69, 925-937.

Kiyonari, T., \& Yamagishi, T. (1999). A comparative study of trust and trustworthiness using the game of enthronement. Japanese Journal of Social Psychology, 15, 100-109.

Macy, M. W., \& Skvoretz, J. (1998). The evolution of trust and cooperation between strangers: A computational model. American Sociological Review, 63, 638-60.

Markus, H. R., \& Kitayama, S. (1991). Culture and the self: Implications for cognition, emotion, and motivation. Psychological Review, 98, 224-253.

Pratt, M. W., Prancer, M., Hunsberger, B., \& Manchester, J. (1990). Reasoning about the self and relationships in maturity: An integrative complexity analysis of individual differences. Journal of Personality and Social Psychology, 59, 575-581.

Seeley, E. A., Gardner, W. L., Pennington, G., \& Gabriel, S. (2003). Circle of friends or members of a group? Sex differences in relational and collective attachment to groups. Group Processes $\mathcal{E}$ Intergroup Relations, 6, 251-263.

Sorrentino, R. M., Hewitt, E. C., \& Raso-Knott, P. A. (1992). Risk-taking in games of chance and skill: Informational and affective influences on choice behavior. Journal of Personality and Social Psychology, 62, 522-533.
Weber, E. U., Blais, A., \& Betz, N. E. (2003). A domain-specific risk-attitude scale: Measuring risk perceptions and risk behaviors. Journal of Behavioral Decision Making, 15, 263-290.

Yamagishi, T., \& Kiyonari, T. (2000). The group as the container of generalized reciprocity. Social Psychology Quarterly, 63, 116-132.

Yamagishi, T., \& Yamagishi, M. (1994). Trust and commitment in the United States and Japan. Motivation and Emotion, 18, 129-166.

Yuki, M. (2003). Intergroup comparison versus intragroup cooperation: A cross-cultural examination of social identity theory in North American and East Asian cultural contexts. Social Psychology Quarterly, 66, 166-183.

Yuki, M., Maddux, W. W., Brewer, M. B., \& Takemura, K. (2005). Cultural differences in relationship- and group-based trust. Personality and Social Psychology Bulletin, 31, 48-62.

Zuckerman, D. M. (1985). Confidence and aspirations: Self-esteem and self-concepts as predictors of students' life goals. Journal of Personality, 53, 543-560.

Paper received 12 March 2004; revised version accepted 30 September 2004.

\section{Biographical notes}

WILliam W. MADDUX is a Visiting Assistant Professor of Management \& Organizations, and a Post-Doctoral Fellow at the Dispute Resolution Research Center, Kellogg School of Management, Northwestern University.

MARILYNN B. BREWER is a Professor of Psychology, and the Ohio State Eminent Scholar in Social Psychology at the Ohio State University. 